\title{
Characterization of surface Ag nanoparticles in nanocomposite a-C:Ag coatings by grazing incidence $X$-ray diffraction at sub-critical angles of incidence
}

\author{
N. K. Manninen ${ }^{1} \cdot$ J. C. Oliveira ${ }^{1} \cdot$ S. Carvalho $^{2} \cdot$ A. Cavaleiro $^{1}$
}

Received: 4 August 2015/Accepted: 13 October 2015/Published online: 17 February 2016

(C) Springer-Verlag Berlin Heidelberg 2016

\begin{abstract}
Silver diffusion within nanocomposite films and/or toward the film surface is often observed during annealing of the silver-based nanocomposite films. In order to control and/or minimize this process, it is crucial to characterize the aggregated silver nanoparticles on the films surface. In this paper grazing incidence X-ray diffraction (GIXRD) with both sub-critical and supra-critical angles of incidence is used to characterize the $\mathrm{Ag}$ nanoparticles distribution, shape and structure both inside the matrix and on the nanocomposite film surface. The nanocomposite carbon coating containing Ag nanoparticles (a-C:Ag) was deposited by dc magnetron sputtering. The coatings were analyzed by GIXRD using fixed incident angles both below and above the critical angle for total reflection. By using sub-critical angles it was possible to eliminate diffraction from the bulk material allowing to estimate the size distribution of the nanoparticles sitting on the surface. The results obtained by GIXRD analysis were checked through comparison with the observations made by both TEM and SEM analysis. The proposed methodology can be used to characterized nanoparticles deposition on a surface and/or island formation during film growth as long an adequate substrate with high critical angle for total reflection is used.
\end{abstract}

N. K. Manninen

nora.sousa@dem.uc.pt

1 SEG-CEMUC, Mechanical Engineering Department, University of Coimbra, 3030-788 Coimbra, Portugal

2 GRF-CFUM, Physics Department, University of Minho, 4800-058 Guimarães, Portugal

\section{Introduction}

Nanocomposite thin films containing Ag have been proposed and evaluated for a large variety of applications due to their interesting optical $[1,2]$, electrical $[2,3]$ and tribological/mechanical [4-6] properties as well as their biological activity $[7,8]$. Besides the specific properties of $\mathrm{Ag}$ and the matrix material, the performance of the nanocomposite thin films critically depends on the $\mathrm{Ag}$ nanoparticles size distribution and morphology as well as on the interfaces established between the nanoparticles and the matrix. Accordingly, a careful characterization of the nanocomposite films morphology and nanostructure is the first step toward the understanding of the film properties evolution and subsequent optimization of its performance.

Microscopy-based characterization techniques, such as transmission electron microscopy (TEM), scanning electron microscopy (SEM) and scanning tunneling microscopy (STM), have become very popular, since they allow the direct observation of nanoparticles morphology and size distribution. On the other hand, scattering techniques, such as X-ray diffraction (XRD) and small angle X-ray scattering (SAXS), have also been largely used for evaluating the nanoparticles size distribution and crystalline structure, allowing to obtain a more accurate statistical analysis in case of heterogeneous samples [9-14]. Regarding X-ray diffraction, the most useful configuration for thin film analysis is grazing incidence $\mathrm{X}$-ray diffraction (GIXRD). In this configuration the path travelled by X-rays within the thin film is increased as compared to symmetric geometries, such as the Bragg-Brentano configuration, while the penetration depths of the X-rays remain closer to the film thickness. As a result, the interaction of the X-rays with the film material is maximized allowing to acquire more detailed information about the film while minimizing 
the possible interference of the substrate. As shown in Fig. 1, in GIXRD a parallel monochromatic X-ray beam impinges on the surface at a fixed incidence angle $(\alpha)$, with a value higher but close to the critical angle for total external reflection, $\alpha_{c}$, while the detector is rotated on a goniometer circle. However, useful information about the thin films can also be obtained by using sub-critical angles of incidence. Below (typically $80 \%$ ) the critical angle of the surface material studied, an evanescent wave is established for a short distance and is exponentially damped. Therefore, Bragg reflections are only coming from the surface structure $[15,16]$.

Silver diffusion within nanocomposite films and/or toward the film surface is often observed during annealing of the silver-based nanocomposite films [2, 4, 5, 17, 18]. Manninen et al. [18] reported that these phenomena also occur at room temperature if an adequate period of time is given. Silver diffuses to the film surface where it aggregates in a wide variety of shapes and forms depending on the specific experimental conditions $[4,5,18]$. Silver diffusion critically affects the nanocomposite films properties and its performance with time. In most cases, this is an unwanted effect as the optimized initial Ag nanoparticles size distribution and morphology are lost with time. However, in some cases, this effect can be an advantage allowing, for example, controlled released of silver for biomedical applications. In order to control and/or minimize this process, it is crucial to characterize the aggregated silver nanoparticles on the films surface. Although microscopy-based techniques allow direct observation of these nanoparticles, they have to be complemented with characterization techniques allowing a more accurate statistical analysis such as SAXS. However, SAXS is a complex technique which uses synchrotron radiation which is not readily available. On the other hand, it does not allow to characterize the structure of the aggregated Ag nanoparticles.

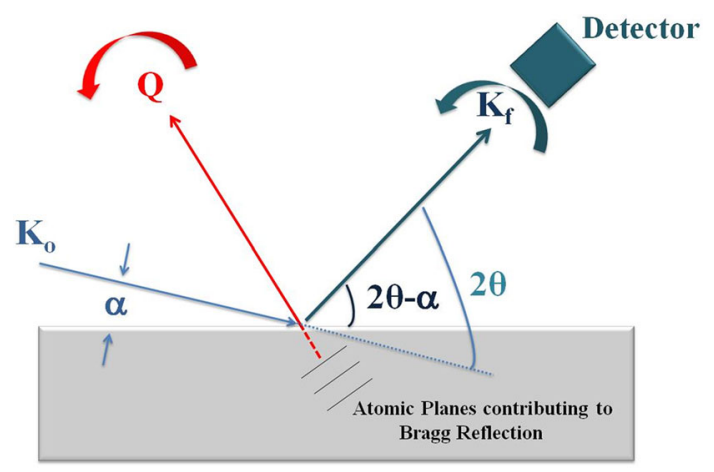

Fig. 1 Schematic representation of the diffractometer setup used in GIXRD measurement. $Q$ is the scattering plane, $K_{o}$ the incident vector, $K_{f}$ the diffracted vector and $\alpha$ the incidence angle
In this paper GIXRD using both sub-critical and supracritical angles of incidence is used to characterize the $\mathrm{Ag}$ nanoparticles distribution, shape and structure both inside the matrix and on the nanocomposite film surface. It is shown that this simple non-destructive technique that does not require previous specimen preparation can be used to gather information of the aggregation state of $\mathrm{Ag}$ on the film surface.

\section{Experimental}

Nanocomposite carbon coatings containing Ag nanoparticles (a-C:Ag) were deposited by dc magnetron sputtering onto single crystalline silicon (100) and TEM copper grids with carbon foil substrates. Power densities of 7 and $0.09 \mathrm{~W} / \mathrm{cm}^{2}$ were applied to the pure $\mathrm{C}$ and $\mathrm{Ag}$ targets $(99.99 \%, 200 \times 100 \times 10 \mathrm{~mm})$, respectively. All deposition was carried out in pure Ar discharge gas at $0.5 \mathrm{~Pa}$, while the rotating substrates $(18 \mathrm{rpm})$ were placed at $10 \mathrm{~cm}$ from both targets. A pulsed negative dc bias voltage of $50 \mathrm{~V}$ was applied to the substrate holder which was not intentionally heated. The deposition time was adjusted to obtain coatings with a thickness of 750 and $40 \mathrm{~nm}$ for GIXRD and TEM analysis, respectively.

The chemical composition coating was determined by electron probe microanalysis (EPMA), with a Cameca SX 50 apparatus; five punctual measurements were randomly performed on samples surface immediately after deposition, with an acceleration voltage of $10 \mathrm{kV}$. The EPMA results indicate that the coatings contain 20 at. $\%$ of $\mathrm{Ag}$. The GIXRD experiments were carried out in a PANalytical X'Pert PRO MPD system using $\mathrm{Cu} \mathrm{K} \alpha$ radiation $(45 \mathrm{kV}$ and $40 \mathrm{~mA}$ ) with a parallel-beam configuration. The incident beam optics consisted of a hybrid monochromator [with a $\mathrm{Cu}$ W/Si mirror and a double crystal Ge (220)]. A parallel plate collimator $\left(0.7^{\circ}\right)$ and Soller slits $\left(0.004^{\circ}\right)$ were mounted on the path of the diffracted beam. A PIXcel detector in receiving slit mode was used for X-ray collection. TEM and SEM analysis were performed in Tecnai G2 working at $200 \mathrm{kV}$ and EDAX Nova nanoSEM 200, respectively.

\section{Results and discussion}

The GIXRD patterns of the deposited nanocomposite film obtained at different incident angles are shown in Fig. 2a. In each case the incidence angle was fixed, between 0.1 and $0.7^{\circ}$, while the detector was rotated in a range corresponding to diffraction angles between 30 and $50^{\circ}$. At low incidence angles, below $0.2^{\circ}$, two narrow peaks are detected at $2 \theta$ values slightly higher than the Ag (111) and 
(a)
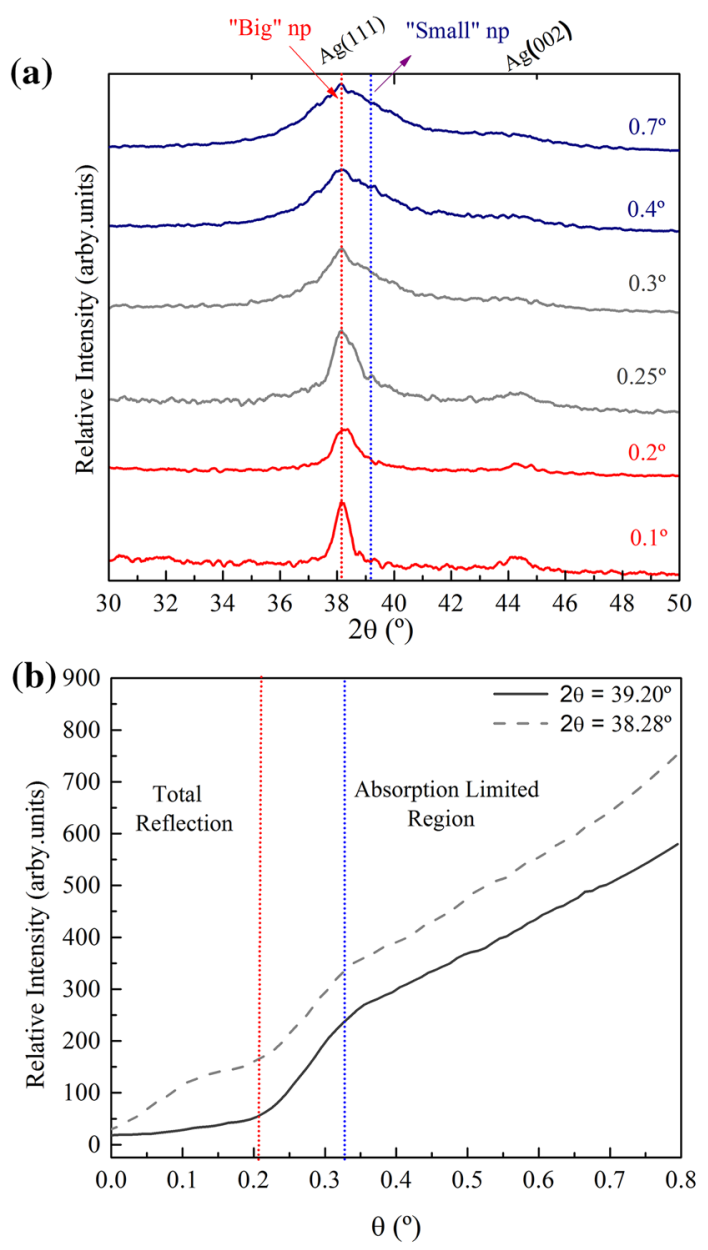

(c)

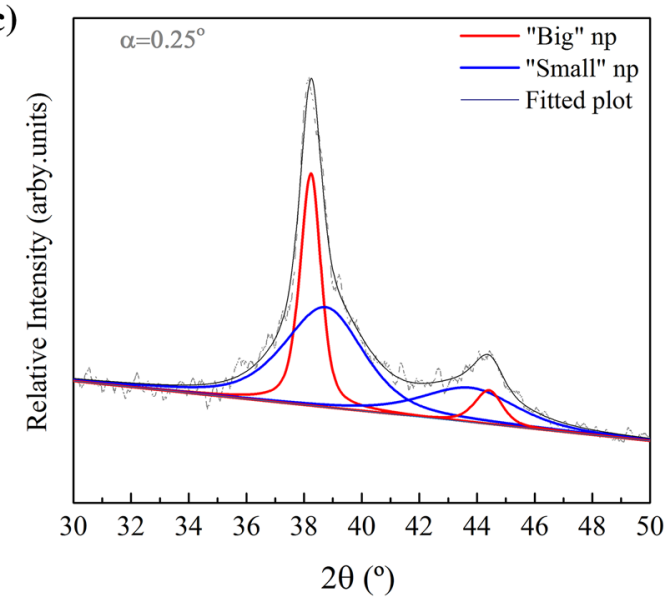

Fig. 2 a GIXRD patterns obtained at different grazing angles, b variation of diffraction peaks intensity at different grazing angles $\left(2 \theta=39.20^{\circ}\right.$ and $\left.2 \theta=38.28^{\circ}\right)$ and $\mathbf{c}$ fitting of GIXRD pattern obtained at $\alpha=0.25$

Ag (200) diffraction angles in the Ag ICCD card. As the incident angle is increased above $0.2^{\circ}$, a broader diffraction peak starts to appear in the high angle side of each of the peaks detected at lower incident angles. The intensity of the broad peaks increases with increasing incident angle resulting in an increasing asymmetry and progressive broadening of the overall GIXRD peaks with increasing incident angle. Both sets of peaks correspond to $\mathrm{Ag}$ (111) and (200) diffraction peaks although the large difference in the FWHM shows that they are originated from Ag phases with different microstructures. In order to identify the origin of the XRD peaks, the intensity of the GIXRD diffraction was recorded at $2 \theta=38.28^{\circ}$ and $39.20^{\circ}$ as a function of the incident angle (Fig. 2b). In the former case, the detector was fixed at the position corresponding to the Ag (111) narrow peak recorded at low incidence angle in Fig. 1. In the latter case the detected diffraction signal corresponds to the $\mathrm{Ag}$ (111) broad peak recorded at higher incident angle in the same figure. The evolution of the diffraction intensity corresponding to the broad Ag (111) peak follows the expected behavior for a homogeneous carbon layer which has a critical angle near $0.2^{\circ}$. The intensity of the diffraction signal is very weak below the critical angle as the penetration depth is limited to only a few atomic layers and total external reflection of the X-ray occurs. As the incident angle increases above the critical angle, an abrupt increase in the XRD signal is observed as the depth of penetration of the X-rays in the material increases exponentially. At higher incident angles (above $0.4^{\circ}$ ), the XRD depth of penetration increases until the absorption limited regime is achieved. The increasing intensity of the detected X-ray diffraction signal in this regime is due to the measuring setup used in this work. At low incidence angles the X-ray beam is spread on the sample surface plane over an area much bigger than the sample size. As the incident angle is increased the spread of the X-ray beam decreases and a higher fraction of the emitted X-ray impinge on the sample surface leading to increasing intensity. The above consideration allows to conclude that the broad Ag diffraction peaks detected at higher incident angle in Fig. 2a originate from the $\mathrm{Ag}$ nanoparticles dispersed inside the carbon matrix in the nanocomposite film. The evolution of the diffracted intensity of the narrow Ag (111) peak, recorded with the detector fixed at $2 \theta=38.28^{\circ}$, also shows the abrupt increase in intensity above the carbon critical angle and the smoother increase at higher incident angles. However, the recorded intensity shows an additional feature: it initially increases up to $0.1^{\circ}$ in such a way that a significant signal intensity is recorded already below the carbon critical angle. As the X-rays are not able to significantly penetrate the nanocomposite film at incident angles below the critical angle (total external reflection regime), the increased intensity below the critical angle has to be due to $\mathrm{Ag}$ on the film surface. The formation of a thin $\mathrm{Ag}$ layer on the nanocomposite film surface, through diffusion of the $\mathrm{Ag}$ nanoparticles dispersed in the carbon matrix, would not 
explained the above results as the Ag critical angle is even higher than that of carbon (near $0.4^{\circ}$ ). However, the formation of $\mathrm{Ag}$ nanoparticles on the film surface should result in diffraction even below $0.2^{\circ}$. Assuming a spherical shape for the Ag nanoparticles, some of the parallel-beam $\mathrm{X}$-rays will always impinge on the nanoparticles at angles above the critical angle of silver. As a result, part of the incident X-ray will be able to penetrate inside the Ag nanoparticles and therefore diffraction will occur.

All the XRD peaks in Fig. 2a were best fitted with pseudo-Voigt functions, and their FWHM was calculated taking into account the instrumental broadening. As an example, the fitting performed for the pattern obtained at $\alpha=0.25^{\circ}$ is shown in Fig. 2c. In this case, two contributions were found for each of the $\mathrm{Ag}$ peaks. The broader contributions, which are also present in the GIXRD patterns obtained at higher incident angles, are attributed to the Ag-np dispersed in the carbon matrix. The narrower contributions, which are the only ones present at incident angles below $0.25^{\circ}$, were attributed to the Ag-np sitting on the film surface. Calculation of the size of both types of Ag-np using the $\operatorname{Ag}(111)$ peak and the Scherer formula [19] showed that the Ag-np dispersed in the carbon matrix is significantly smaller $(2 \mathrm{~nm})$ than those sitting on the film surface $(18.6 \mathrm{~nm})$.

The formation of Ag-np on the surface of Ag-based nanocomposite films with bigger sizes than those present in the bulk was already reported for $\mathrm{TiO}_{2}[2]$ and $\mathrm{SiC} / \mathrm{HfC}$ [4] matrixes. It is mainly attributed to the high mobility of $\mathrm{Ag}$ nanoparticles and the lack of restrictions for the nanoparticles growth on the film surface as compared to the bulk.

The SEM cross-sectional and top-view (inset) micrographs of the a-C:Ag nanocomposite coatings analyzed by GIXRD are shown in Fig. 3a. The cross-sectional micrograph shows that a-C:Ag coating has a compact morphology, but no evidence of $\mathrm{Ag}$ nanoparticles is found. However, the top-view micrograph clearly shows that the coatings surface is covered by approximately spherical Ag nanoparticles. Image analysis using the ImageJ software package revealed that the $\mathrm{Ag}$ nanoparticles are slightly elongated with a mean size of $22 \pm 8 \mathrm{~nm}$ in the longer axis direction and $16 \pm 6 \mathrm{~nm}$ in the shorter axis dimensions. Note that the resolution of the SEM micrograph only allowed to take into account the Ag nanoparticles with sizes above $10 \mathrm{~nm}$ for the statistical analysis. In order to characterize the nanoparticles dispersed in the carbon matrix, the a-C:Ag nanocomposite coatings was analyzed by TEM. The top-view micrograph of the films clearly shows a bi-modal distribution (Fig. 3b): nanoparticles with sizes up to $20 \mathrm{~nm}$ are found together with nanoparticles with sizes under $3 \mathrm{~nm}$. Examples of both types of nanoparticles are underlined in Fig. 3b. The size of bigger nanoparticles is close to the size of the Ag-np detected on
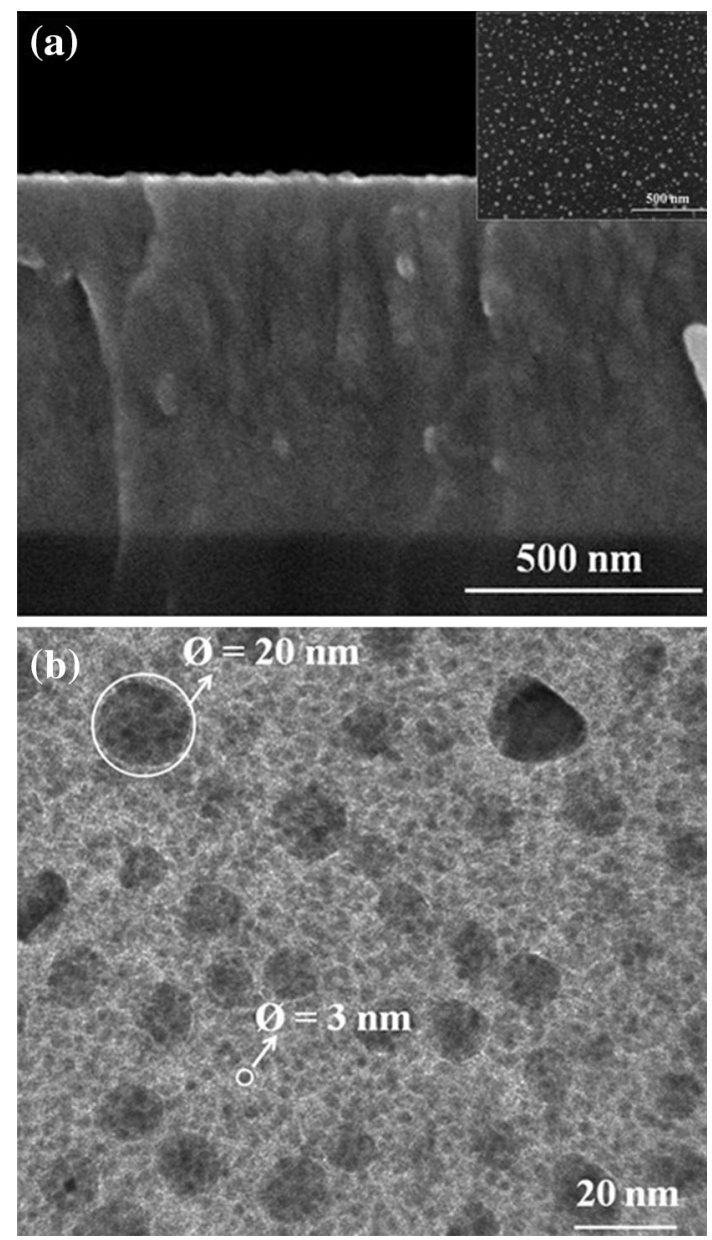

Fig. 3 a SEM cross-sectional and top-view micrograph (inset) and b TEM top-view micrograph of a-C:Ag nanocomposite coating

the film surface by both SEM and GIXRD $(\approx 18.6 \mathrm{~nm})$. On the other hand, the size of the smaller nanoparticles is close to the size of the Ag-np dispersed inside the carbon matrix already detected by GIXRD $(\approx 2 \mathrm{~nm})$. The microscopy analysis of the nanocomposite film clearly validates both the interpretation and the results obtained by GIXRD.

\section{Conclusion}

In this work it was shown that GIXRD using sub-critical angles of incidence is a suitable technique to characterize nanoparticles sitting on materials surface. By using subcritical angles it is possible to eliminate diffraction from the bulk material, while diffraction from the surface nanoparticles still occurs. The resulting diffractograms can be used to estimate the size distribution of the nanoparticles sitting on the surface and to study there structure without interference from the bulk material. In the present work, the size of the Ag nanoparticles sitting on the surface of the 
nanocomposite Ag-based film (20 nm) was chosen in order to allow direct comparison with the SEM analysis. However, the present methodology can be used to study smaller nanoparticles which could only be analyzed by TEM. The main advantage of the GIXRD technique is that it can be readily applied to a large amount of coatings without the need of time consuming specimen preparation as in TEM. On the other hand, it is a non-destructive and quite fast characterization technique. Although a parallel-beam diffraction configuration is required for GIXRD, in order to avoid dispersion of the incident angle on the film surface, this kind of setup is nowadays readily available in many diffraction laboratories. Finally, it is worst to point out that the methodology used in this work can be used to characterized nanoparticles deposition on a surface and/or island formation during film growth as long an adequate substrate with high critical angle for total reflection is used.

Acknowledgments We gratefully acknowledge the financial support provided by the FCT-Fundação para a Ciência e Tecnologia and FSE for the grant SFRH/BD/82472/2011. This research is sponsored by the FEDER funds through the program COMPETE-Programa Operacional Factores de Competitividade and by the national funds through FCT-Fundação para a Ciência e Tecnologia in the framework of the Strategic Projects PEST C/EME/UIO0285/2011.

\section{References}

1. X. Yang, W. Li, Z. Li, Y. We, W. Huang, Appl. Phys. A 90, 465-467 (2008)
2. V.S.K. Chakravadhanula, C. Kubel, T. Hrkac, V. Zaporojtchenko, T. Strunskus, F. Faupel, L. Kienle, Nanotechnology 23, 495701 (2012)

3. S.M. Marques, N.K. Manninen, S. Ferdov, S. Lanceros-Mendez, S. Carvalho, Appl. Surf. Sci. 317, 490 (2014)

4. E. Kzranowski, J.L. Endrino, J.J. Nainaparampil, J.S. Zabinski, J. Mater. Eng. Perfor. 13(4), 439 (2004)

5. C.P. Mulligan, T.A. Blanchet, D. Gall, Wear 269, 125 (2010)

6. I. Ferreri, V. Lopes, S. Calderon, C.J. Tavares, A. Cavaleiro, S. Carvalho, Mater. Sci. Eng. C 42, 790 (2014)

7. J.L. Endrino, J.C. Sanchéz-Lopez, R.E. Galindo, D. Howart, A. Anders, J. Phys.: Conf. Series 252, 012005 (2010)

8. A. Parveen, T. Ali, M. Wahid, S. Rao, Matter. Lett. 148, 86-90 (2015)

9. H. Borchert, E.V. Shevchenko, A. Robert, I. Mekis, A. Kornowski, G. Grubel, H. Weller, Langmuir 21, 1931 (2005)

10. B.D. Hall, D. Zanchet, D. Ugarte, J. App. Crystallog. 33, 1335 (2000)

11. E. Carvou, J.L. Le Garrec, J. Perez, J. Praquin, M. Djeddi, J.B.A. Mitchell, AIP Adv 3, 032139 (2013)

12. B. Abecassis, F. Testard, O. Spalla, P. Barboux, Nano Lett. 7, 1723 (2007)

13. B. Chu, T. Liu, J. Nanop. Res. 2, 29 (2000)

14. K. Koga, H. Takeo. Rev. Sci. Instrum. 67, 4092 (1996)

15. M. Birkholz, Thin Film Analysis by X-Ray Scattering (Wiley$\mathrm{VCH}$, Singapore, 2006)

16. M.F. Toney, S. Bernnan, J. App. Phy. 65, 4763 (1989)

17. R.E. Galindo, N.K. Manninen, C. Palacio, S. Carvalho, Anal. Bioanal. Chem. 405, 6259 (2013)

18. N.K. Manninen, R.E. Galindo, S. Carvalho, A. Cavaleiro, Surf. Coat. Technol. 267, 90-97 (2015)

19. P. Scherrer, N.G.W. Gottingen, Math. Phys. Kl. 2, 96 (1918) 